\title{
Increasing Accuracy of Atrophy Measures from Serial MR Scans Using Parameter Analysis of the Boundary Shift Integral
}

\author{
Richard G. Boyes, Jonathan M. Schott, Chris Frost, and Nicholas C. Fox \\ Dementia Research Group, Institute of Neurology, University College London, \\ 8-11 Queen Square, London WC1N 3BG, UK
}

\begin{abstract}
A statistical method is proposed to determine ideal window parameters for the boundary shift integral (BSI), based on comparing the BBSI to segmented volume differences for a range of windowing parameters. Upon application, new parameters were obtained to measure brain atrophy in 35 subjects (23 AD, 12 controls), and group separation measured. Group separation increased and the numbers required to power a treatment trial decreased.
\end{abstract}

\section{Introduction}

The boundary shift integral (BSI) is a measure of cerebral atrophy from serial MRI. It requires two parameters which specify the location and width of a sampling window. The BSI increases the precision of estimating atrophy (compared to segmented volume differences (SVDs)) but may slightly underestimate atrophy. We assumed we could choose parameters that would approximate the BSIs to the SVDs without reducing precision for a set of Alzheimer's disease (AD) and control subjects.

\section{Methods}

Twenty-three patients with AD and twelve age-matched controls had two volumetric T1-weighted MR scans acquired approximately one year apart. The repeat scan was rigidly registered brain-to-brain to the baseline.

The BSI [1] measures brain atrophy between a co-registered scan pair. It is calculated by subtracting normalised intensities between the borders of the coregistered brains to give a volume. $I_{c}$ (window centre) and $I_{w}$ (window width) are user defined normalised intensity parameters; they define an intensity window such that any intensity above or below the bounds of the window is set to the upper or lower bound, respectively.

In order to determine improved values of $I_{c}$ and $I_{w}, t$-tests were used to assess the difference between volumes of the SVDs and BSIs (which should approximate each other) for all co-registered scans. Pitman's test was used to assess the difference in variance (BSI variance should be lower). The variability of these 
test statistics over the range of $I_{c}$ and $I_{w}$ allowed us to estimate evidence for differences in means or variances, and hence chose an improved $I_{c}$ and $I_{w}$.

Changes in volume were calculated using the BSI and expressed as an annual $\%$ loss using the chosen parameters, group separation measured and power calculations [2] carried out for the original $\left(I_{c}=0.5, I_{w}=0.5\right)$ and new parameters.

\section{Results and Discussion}

We chose an $\left(I_{c}, I_{w}\right)$ of $(0.6,0.2)$ because an $I_{c}$ of 0.6 gave a close approximation to the SVDs (left figure) but also decreased BSI variability (right figure). An $I_{w}$ of 0.2 was used as a trade off between the gain of the BSI and robustness.
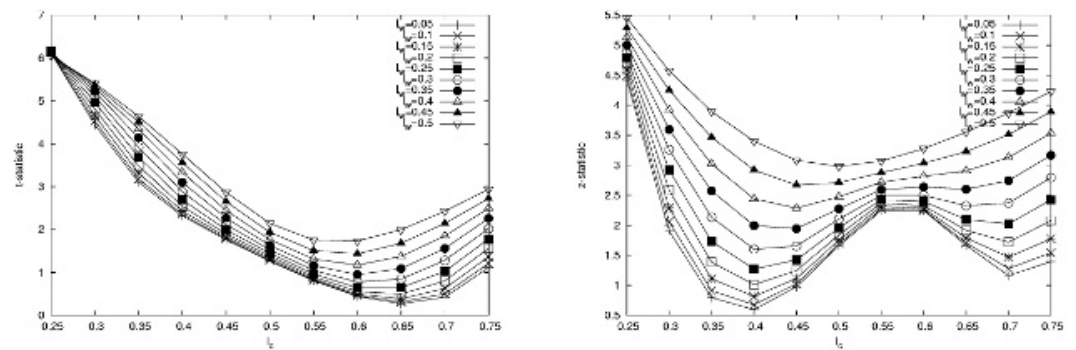

Using these parameters and comparing them to $\left(I_{c}, I_{w}\right)=(0.5,0.5)$ the mean atrophy rate increased from $1.98 \pm 0.83$ to $2.27 \pm 0.83$ for AD subjects, while controls increased to a lesser extent, from $0.48 \pm 0.46$ to $0.50 \pm 0.46$. Some evidence $(p=0.06)$ (using an unpaired $t$-test with unequal variances) was found that group separation had increased.

Applying methods for estimating numbers required to power a drug trial [2] for both old and new BSIs, for a drug anticipated to reduce excess atrophy by $20 \%$ over one year with $10 \%$ dropout, $22 \%$ fewer subjects would be required to achieve $90 \%$ statistical power for the new parameters.

By altering the parameters of the BSI, we have demonstrated that it is possible to increase the accuracy of whole brain atrophy measurements. The alteration in atrophy rates derived using this method in patients with $\mathrm{AD}$ and controls has clinical relevance: the numbers of patients required in trials of putative diseasemodifying treatments are reduced, in this case by over $20 \%$.

\section{References}

1. Freeborough, P.A., Fox, N.C.: The boundary shift integral: an accurate and robust measure of cerebral volume changes from registered repeat MRI. IEEE TOMI 16 (1997) 623-629

2. Fox, N.C., Cousens, S., Scahill, R.: Using serial registered brain magnetic resonance imaging to measure disease progression in Alzheimers Disease: Power calculations and estimates of sample size to detect treatment effects. Arch. Neurol. 57 (2000) $339-344$ 